OPEN ACCESS

Edited by:

Julio de la Torre,

Comillas Pontifical University, Spain

Reviewed by:

Marija Ban,

University Hospital Split, Croatia

Francesco Pepe,

University of Naples Federico II, Italy

Marco Invernizzi,

University of Eastern Piedmont, Italy

*Correspondence:

Angeline Ginzac

angeline.ginzac@clermont.unicancer.fr

Specialty section: This article was submitted to

Breast Cancer,

a section of the journal

Frontiers in Oncology

Received: 20 July 2021 Accepted: 24 November 2021 Published: 13 December 2021

Citation:

Ginzac A, Bernadach M, Molnar I, Duclos M. Thivat $E$ and Durando X (2021) Adapted Physical Activity for Breast Cancer Patients Treated with Neoadjuvant Chemotherapy and Trastuzumab Against HER2 (APACAN2): A Protocol for a Feasibility Study.

Front. Oncol. 11:744609. doi: 10.3389/fonc.2021.744609

\section{Adapted Physical Activity for Breast Cancer Patients Treated with Neoadjuvant Chemotherapy and Trastuzumab Against HER2 (APACAN2): A Protocol for a Feasibility Study}

\author{
Angeline Ginzac ${ }^{1,2 *}$, Maureen Bernadach ${ }^{3,4}$, loana Molnar ${ }^{1,2}$, Martine Duclos $^{5}$, \\ Emilie Thivat ${ }^{1,2}$ and Xavier Durando ${ }^{1,4}$
}

\begin{abstract}
1 INSERM U1240 IMOST, Université Clermont Auvergne, Clermont-Ferrand, France, ${ }^{2}$ Département de Recherche Clinique, Délégation Recherche Clinique et Innovation, Centre Jean PERRIN, Clermont-Ferrand, France, ${ }^{3}$ Centre d'Investigation Clinique UMR501, Clermont-Ferrand, France, ${ }^{4}$ Service d'Oncologie Médicale, Centre Jean PERRIN, Clermont-Ferrand, France, ${ }^{5} \mathrm{CHU}$ Clermont-Ferrand, Service de Médecine du Sport et des Explorations Fonctionnelles, Université Clermont Auvergne, INRAE, UNH, Clermont-Ferrand, France
\end{abstract}

Background: The standard care for HER2-positive breast cancer is chemotherapy plus a HER2-directed therapy. This can lead to treatment-induced cardiotoxicity. On the other hand, the practice of physical activity is known to improve cardiac function; thus HER2positive breast cancer patients could draw particular benefit from physical activity during treatment. However, at the time of diagnosis for breast cancer, the majority of patients are insufficiently active according to physical activity recommendations of World Health Organisation, and it is difficult to remain or become active during the treatment. There is a lack of data in the literature on the optimal program to propose to patients to encourage them to be active during treatment. The aim of our study is to assess the feasibility of a home-based physical activity program during neoadjuvant chemotherapy and trastuzumab for HER2-positive breast cancer.

Methods: The APACAN2 study is a single-centre, non-randomized interventional trial. Patients with HER2-positive breast cancer treated with anthracycline-based neoadjuvant chemotherapy and trastuzumab are eligible for enrolment. The supervised home-based physical activity program takes place during neoadjuvant chemotherapy (NACT). It combines aerobic and strengthening exercises. The primary endpoint is the proportion of patients reaching the international physical activity recommendations, i.e. 150 minutes of moderate-intensity activity per week at the end of NACT. The study started in April 2018 and seventy patients are expected to be recruited.

Discussion: In the literature, the majority of studies on practice of physical activity in breast cancer focus on adjuvant chemotherapy or on the period after the end of 
treatment. To the best of our knowledge, the APACAN2 study is the first to evaluate a home-based physical activity program during neoadjuvant chemotherapy for HER2positive breast cancer.

Trial Registration Number: Clinicaltrials.gov: NCT02963363, registered on July 11, 2016. Identifier with the French National Agency for the Safety of Medicines and Health Products N'ID RCB 2016-A01344-47, registered in August 2016. Protocol: version 8, 24 February 2021.

Keywords: HER2-positive breast cancer, adapted physical activity, neoadjuvant chemotherapy, HER2-directed therapy, feasibility study

\section{INTRODUCTION}

Worldwide, breast cancer is the most commonly diagnosed cancer among women and is also the leading cause of cancer death (1). Overexpression of human epidermal growth factor receptor 2 (HER2) concerns approximately 15\% of breast cancer patients. This subtype of cancer is associated with a poorer disease-free survival and overall survival $(2,3)$. The standard care to treat these tumours is chemotherapy plus HER2-directed therapy administered in combination with standard chemotherapy (anthracycline and taxane-based). This treatment reduces both the rate of recurrence and the mortality rate, respectively by half and by one third. However, trastuzumab (the most commonly used HER2-directed therapy) is known to be associated with cardiotoxicity (4). Anthracycline-based chemotherapy also causes cardiotoxicity $(5,6)$. Between 1 and $4 \%$ of patients treated with trastuzumab develop heart failure is and nearly $10 \%$ have asymptomatic LVEF reduction (7-10). It has been estimated that the cumulative incidence rate for major cardiac events (as for example pulmonary edema, cardiomyopathy; cardiovascular death) is $6.6 \%$ for sequential therapy, i.e. anthracycline and trastuzumab (11).

As a result, HER2-positive breast cancer patients are exposed to treatment-induced cardiotoxicity. This can have serious consequences, such as treatment discontinuation (12).

Physical exercise is known to improve quality of life during and after breast cancer treatment (13-16). It can also be beneficial for cardiac function. Eight weeks of aerobic practice during adjuvant chemotherapy for breast cancer have led to an increase in oxygen peak consumption reflecting an improvement in cardiorespiratory capacities (17). The longer the physical activity program, the greater the improvement in oxygen peak consumption (ranging from $0.6 \mathrm{ml} / \mathrm{min} / \mathrm{kg}$ after 12 weeks to $1.83 \mathrm{ml} / \mathrm{min} / \mathrm{kg}$ after 27 weeks of practice) (18-20). In addition, physical activity could protect the cardiovascular system, so that it could be a strategy to limit treatment-induced cardiotoxicity in the HER2-positive population. However, at diagnosis of breast cancer,

Abbreviations: APA, Adapted physical activity; CI, confidence interval; HDL, High density lipoprotein; HER2, Human epidermal growth factor receptor 2; LDL, Low density lipoprotein; LVEF, Left ventricular ejection fraction; MFI-20, Multidimensional fatigue inventory; MSE, Medico-sports educator; NACT, Neoadjuvant chemotherapy; QLQ-C30, Quality of life questionnaire core 30; QOL; Quality of life; RPAQ, Recent physical activity questionnaire. the majority of patients have insufficient levels of physical activity and do not reach the international recommendations (21-25). These recommendations are issued by the World Health Organisation (WHO) for the healthy population, and correspond to 150 minutes of moderate-intensity activity or 75 minutes of vigorous activity or an equivalent combination of moderate and vigorous activity per week (26) and to limit time spent in sedentary behaviors. Nevertheless, it can be difficult to maintain physical activity during treatment because of several barriers relating to care, and also personal barriers (27).

Numerous interventional clinical trials have been set up to promote adapted physical activity during breast cancer treatment. The notion of adapted physical activity refers to physical activities and sports that are adapted to the capacities of people because of their health condition. The proposed programs are heterogeneous in terms of exercises (aerobic and/ or muscular strengthening), duration of sessions, duration of the program (a few weeks to several months), intensity or even the mode of practice (alone or in a group, supervised or not) (27).

In the literature data, there are few trials exploring the interest in physical activity for the HER2-positive breast cancer subpopulation. Haykowski's study (aerobic exercise, three sessions of 30 to 60 minutes/week) concerned the first four months of trastuzumab administration and did not evidence any improvement in cardiac function (28). On the contrary, left ventricular cavity dilation and significant decreases in the ventricular ejection fraction were observed. The authors pointed out that the poor adherence to exercise sessions could explain this result, because cardiopulmonary function improved for patients that completed $\geq 55 \%$ of the sessions. A randomized controlled study among HER2-positive patients showed that patients who followed the aerobic intervention during their neoadjuvant chemotherapy (3 sessions per week, one-to-one supervised sessions) improved their cardiopulmonary function while it decreased in the control group patients (29). In France, the CARDAPAC phase II study conducted on HER2-positive patients treated with trastuzumab alone has just been completed (30). The aim was to assess the impact of 3 months' aerobic exercise on cardiac function and on the incidence of cardiotoxicity. The program started at the end of chemotherapy and consisted in 3 sessions of 45 minutes a week.

The practice of physical activity is recommended as soon as possible at the beginning of treatment for breast cancer patients (31-33). However, in the literature, the majority of the programs 
are offered in the course of adjuvant chemotherapy or in the post-treatment period. Furthermore, there is a lack of information in the literature about the interest of physical activity practice for HER2-positive breast cancer patients. In this context, Jean PERRIN Centre has initiated a prospective interventional study in order to study the feasibility of a homebased physical activity intervention among HER2-positive breast cancer patients currently treated with neoadjuvant chemotherapy + targeted therapy. The objective of the intervention is to achieve or maintain a physical activity level corresponding to the WHO international recommendations at the end of chemotherapy and to limit time spent in sedentary behaviors.

\section{METHODS AND ANALYSIS}

\subsection{Study Design}

APACAN2 is a French single-centre, prospective, interventional, non-randomized trial designed to assess the feasibility of a homebased adapted physical activity (APA) intervention during neoadjuvant chemotherapy for early HER2-positive breast cancer (NCT02963363).

Seventy patients are expected to be recruited. Patient enrolment is expected to take 6 years and the study duration for each patient is 20 months.

Participants can withdraw at any time. Data obtained will be retained with consent, and any reasons given for withdrawal will be recorded.

\subsection{Coordination}

The Centre Jean PERRIN is the sponsor and is responsible for coordination, trial management, data management and trial monitoring.

\subsection{Study Objectives and Endpoints}

\subsubsection{Primary Objective and Endpoint}

The primary objective of the APACAN2 trial is to demonstrate the feasibility of a home-based adapted physical activity (APA) program for patients with HER2 positive breast cancer receiving neoadjuvant chemotherapy. Therefore, the primary endpoint is the proportion of patients reaching the international recommendations for physical activity at the end of chemotherapy. It will be evaluated using the recent physical activity questionnaire (RPAQ) which will be completed before and at the end of neoadjuvant chemotherapy (34).

\subsubsection{Secondary Objectives and Endpoints}

The secondary objectives of the APACAN 2 trial are:

- assessment of the impact of the APA program on exercising time and sedentary time; quality of life; fatigue; weight; physical capacities; physical activity in the rest of the day; lipid profile; ventricular ejection fraction. This will be evaluated using questionnaires [RPAQ (34), QLQ-C30 (35), MFI-20 (36)], physical tests, blood tests and cardiac ultrasound. This data will be collected at baseline, at the end of chemotherapy and at the beginning and the end of targeted therapy.

- description of the longitudinal evolution of physical activity and sedentary behaviour at each step of treatment,

- exploration of barriers to program uptake,

- conditions of the return to work,

- exploration of changes in cancer treatment.

\subsection{Study Procedures and Participant Timeline}

An overview of the study assessments and procedures is presented in Table $\mathbf{1 .}$

Four visits are follow-up appointments for each enrolled patients: inclusion [before the beginning of neoadjuvant chemotherapy (NACT)], at the end of NACT, at the beginning and at the end of HER2-targeted therapy. The study layout is presented in Figure 1.

\subsection{Measures at Each of the Four Assessments}

\subsubsection{Physical Activity and Sedentariness}

The assessment of physical activity and sedentariness will be performed using the modified RPAQ. This questionnaire covers four areas of activity: domestic activities, leisure activities, professional activities, and modes of travel. This questionnaire provides information on the time spent on different activities in each of the four areas mentioned above. Specific energy expenditure is associated with each physical activity, which makes it possible to estimate the overall energy expenditure of patients using Ainsworth compendium (37). It also provides information on the time spent sedentary (work, leisure, travel).

\subsubsection{Measurement of Muscle Strength and Muscular Endurance}

An isokinetic dynamometer will be used to measure the maximum knee extension torque. Subjects will perform the movement with their non-dominant leg (which will be determined as the leg not used to kick a ball). Measurements will be made at different speeds (30,60 and 120\%). For each speed, two trials of 3 successive repetitions will be performed and the best performance will be kept as the maximum isokinetic torque at a given speed. The subjects will have 2 minutes of rest between each trial. Finally, the maximum isometric knee extension torque will be measured at a $120^{\circ}$ angle. Subjects will perform a maximal contraction over 5 seconds or until the isometric torque trace peaks. Subjects will be given 3 trials and 3 minutes of rest between each trial. The best trial will be considered the maximum isometric knee extension torque.

For the upper limbs, muscular strength will be measured with both right and left arm using a dynamometer (hand grip Takei TK 200). Measures will be repeated three times and the best score for each side is retained.

To measure muscular endurance of the lower limbs, the objective for the patient is to maintain a level of force corresponding to $75 \%$ of her maximum voluntary force for as long as possible. 
TABLE 1 | Study timeline.

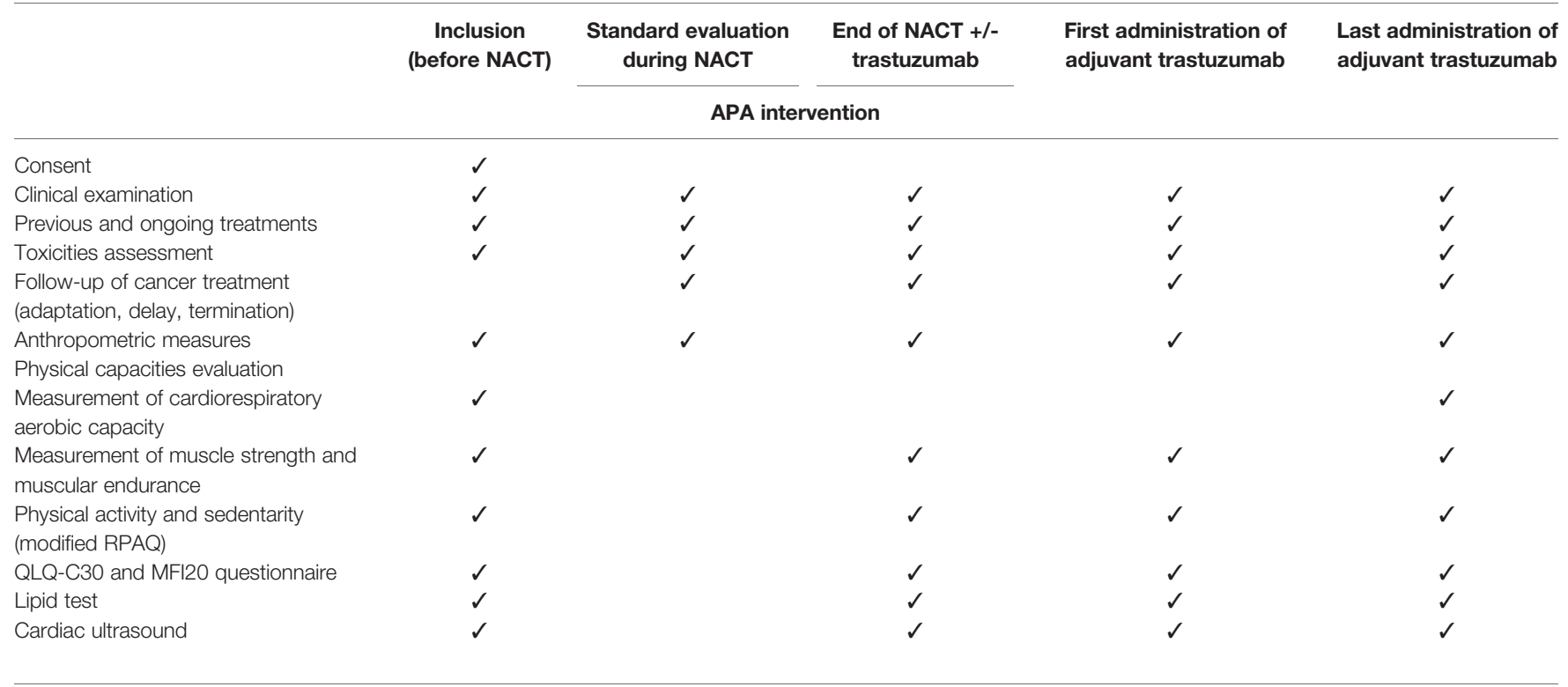

APA, Adapted physical activity.

NACT, Neoadjuvant chemotherapy.

$R P A Q$, Recent Physical Activity Questionnaire.

QLQ-C30, Quality of Life Questionnaire - Core 30.

MFI-20, Mutidimensional Fatigue Inventory.

\subsubsection{Measurement of Cardiorespiratory Aerobic Capacity}

This measure is not mandatory for all patients in order not to delay the beginning of treatment (not related to primary objective) and is expected to be performed twice during participation in the study: at baseline and at the end of the study.

The subjects performed a progressive cycling test on an electromagnetically braked cycle ergometer (Ergoline, Bitz, Germany) until volitional exhaustion to determine the maximal values of ventilation (VEmax), oxygen uptake (VO2max), carbon dioxide output and respiratory exchange ratio (RERmax) by direct method (Oxycon Pro, JAEGER, Germany). VO2 and VCO2 were measured breath-by-breath through a mask connected to $\mathrm{O} 2$ and
CO analysers (Oxycon Pro-Delta, Jaeger, Hoechberg, Germany). Calibration of gases analysers was performed with commercial gases of known concentration. Ventilatory parameters were averaged every $30 \mathrm{~s}$. Electrocardiogram and heart rate (HR) were measured continuously using 10 precordial electrodes. The first stage of the test lasted $3 \mathrm{~min}$, and the initial power output was 35 W. Power output was then increased by $20 \mathrm{~W}$ every 2 min $30 \mathrm{~s}$. Pedaling rate was maintained at 60 revolutions per minute. Criteria for the achievement of $\mathrm{VO} 2 \mathrm{max}$ were subjective exhaustion the participants' maximal HR (HRmax) was closed to their agepredicted maximum HR (i.e., 220-age \pm 10 beats.min-1) and/or Respiratory Exchange ratio (RER, VCO2/VO2) above 1.02 and/or a plateau of $\mathrm{VO} 2$.

\footnotetext{
planned during the study.
}

FIGURE 1 | Design of the APACAN2 study. The APA program take place from the beginning to the end of neo-adjuvant chemotherapy. Four follow-up visits are 


\subsubsection{6-Minute Walking Test}

The test will be run twice in order to reduce the "first time" effect. This test measures the distance a patient can walk in six minutes and thus allows the evaluation of the global and integrated responses of all systems involved during exercise and is considered a proxy for cardiorespiratory capacity. A score is obtained in meters. This test will be performed following the guidelines of the American Thoracic Society (38)and has been tested and validated in the past (39).

\subsubsection{Self-Administered Questionnaires}

The QLQ-C30 (Quality of Life Questionnaire-Core 30) and the MFI20 (Multidimensional Fatigue Inventory) will enable us to evaluate respectively the patient's quality of life (QoL) and fatigue during the study.

\subsubsection{Anthropometric Measures}

Weight will be measured to within $\pm 0.1 \mathrm{~kg}$ on the same scales. The body mass index will be calculated from this data.

Waist circumference will be measured with a tape measure.

Pulse, blood pressure and temperature will be measured by a nurse at the investigating centre.

\subsubsection{Lipid Test}

A lipid assessment will be performed at inclusion, at the end of neoadjuvant chemotherapy and on the day of the first and last administration of anti-HER2 therapy. This assessment will include the determination of total cholesterol, HDL cholesterol, LDL cholesterol and triglycerides. These parameters give an indication of possible cardiovascular risk factors. It will require the collection of $5 \mathrm{ml}$ of blood.

\subsubsection{Cardiac Ultrasound}

A cardiac ultrasound scan will be performed at each of the four appointments. The ventricular ejection fraction results will be evaluated by a cardiologist and compared in order to assess the effect of the APA program on the impact of treatments on cardiac capacity. The cardiac ultrasound is an essential measure for management of cardiovascular function. The same method will be used for each of the assessments for each patient.

\subsection{Selection Criteria}

The inclusion and non-inclusion criteria are presented in Table 2. Patients will be eligible for the study if they have HER2-positive breast cancer with an indication for treatment with neoadjuvant chemotherapy (anthracycline-based) and targeted therapy. They will be ineligible if they have a contraindication for physical activity, a metastatic cancer or a Karnofsky index $\leq 90 \%$

\subsection{Description of the APA Intervention}

The intervention takes place during the neoadjuvant chemotherapy period, i.e. lasting about 18 weeks. The APA program is composed of aerobic exercises and muscle strengthening. An exercise booklet will explain to the patients the different exercises to perform.

Before the beginning of the program, the medical-sports educator (MSE) will demonstrate every exercise of the booklet to the patients. Thereafter patients will perform the program at home. The MSE will call each patient every week to review the program progress and increase or decrease the intensity of exercise according to the patient's experiences.

The aerobic exercises consist in walking on at least five days a week. The duration of walking sessions should increase every week to reach 30 minutes walking per day.

Three times a week, the patients are to perform muscle strengthening with at least 4 different exercises, to mobilize the lower and upper limbs, among those proposed in the booklet. For each of them, the patient are to perform 3 sets of 8 to 12 repetitions with 1 minute of rest between each set.

Patients are asked note all the exercise sessions they do. They should also mention any other physical activity week by week, such as hiking, gardening or cleaning.

\subsection{Statistical Analysis}

The analysis will be done by the biostatistician of clinical research department in Jean PERRIN Center.

\subsubsection{Sample Size}

Our hypothesis is that at least $65 \%$ of the patients will have reached the physical activity recommendations at the neoadjuvant

TABLE 2 | Selection criteria.

\begin{tabular}{|c|c|}
\hline \multirow[t]{5}{*}{ Inclusion criteria } & - Woman 18 years old and older \\
\hline & - Patient with HER2-positive breast cancer, histologically confirmed, eligible to a neoadjuvant chemotherapy and an anti-HER2 target therapy \\
\hline & - Covered by social security system \\
\hline & - Signed informed consent \\
\hline & - Certificate of non-contraindication to the practice of physical activity \\
\hline \multirow[t]{10}{*}{ Non-inclusion criteria } & - History of cancer in the last five years except basocellular \\
\hline & - Metastatic cancer \\
\hline & - Karnofsky index $\leq 90 \%$ \\
\hline & - Men \\
\hline & - Pregnant women \\
\hline & - Patient with psychiatric or cognitive disorders \\
\hline & - Patient deprived of liberty by judicial or administrative decision \\
\hline & - Contraindication to the practice of physical activity \\
\hline & - Insufficient knowledge or understanding of the French language to fill in self-questionnaires correctly or to answer an interrogation \\
\hline & - Participation to another clinical study with a similar objective \\
\hline
\end{tabular}


post-chemotherapy evaluation. A total of 70 patients is required to have an accuracy of $\pm 10 \%$ with a $95 \%$ confidence interval (CI). Given this CI, the trial will be considered positive if more than $75 \%$ of our population complies with the international recommendations.

\subsubsection{Data Analysis}

\subsubsection{Primary Analysis}

The primary endpoint will be the percentage of individuals with physical activity after neoadjuvant chemotherapy, as assessed by the RPAQ with 150 minutes or more per week of moderate intensity or 75 minutes or more of vigorous intensity endurance activity or an equivalent combination of moderate to vigorous activity per week. The $95 \%$ confidence interval of this percentage will be calculated using Pearson's approximation. If this confidence interval is greater than $65 \%$, the intervention will be considered effective. Its feasibility will then be demonstrated. If it only reaches $65 \%$ but is greater than $50 \%$, the intervention will be considered "questionable" and below this, its effectiveness will be considered nil.

\subsubsection{Secondary Analysis}

To assess the impact of the APA program, a comparative analysis of these before/after changes will be carried out on physical activity (MET-h/week), sedentariness (minutes), QoL (overall score, sub scores), fatigue, weight, physical capacities (endurance, muscular strength, flexibility), lipid balance, and cardiac function. The following common tests will be used: paired Student's t-test, Mann-Whitney U-test, and $\mathrm{Chi}^{2}$ for paired series for qualitative criteria. We will also evaluate the impact of levels of physical activity and compliance with recommendations on these parameters using the following tests: ANOVA, Pearson's correlation or Spearman rank tests depending on whether the distributions are Gaussian and homoscedastic.

The effect of the physical activity levels on compliance to the program will be tested by correlations. The Hryniuk score will be used to explore the treatment changes occurring.

Concerning the resumption of professional activity, a survival curve for time to recovery will be calculated.

Concerning the analysis of the longitudinal evolution of physical activity and time spent in sedentary behaviour, a mixed-model ANOVA will be used.

The evaluation of barriers to physical activity adherence will be carried out using the following tests: ANOVA, Pearson's correlation or Spearman's rank tests depending on whether the distributions are Gaussian and homoscedastic. A logistic regression will test the respective influence of the different factors on whether or not the recommendations are met.

The secondary endpoints will be compared to control data from the Jean Perrin Centre or with data from the literature whenever possible.

The standard significance level $(\mathrm{p}<0.05)$ will be used for these analyses. As this trial is exploratory, no correction for multiple comparisons will be made.

\subsection{Data Management And Monitoring}

The data collected for the trial will be entered on an electronic case report form (eCRF) (EnnovClinical). The people with access to the data will be the investigators, the clinical research associates, the project leader and the biostatistician. They are authorized professionals and are subject to professional secrecy. The investigator will ensure the accuracy, completeness, and reliability of the data recorded (pseudonymized patient data) and the provision of answers to data queries.

Regular monitoring will be carried out by a clinical research associate mandated by the sponsor. The objectives will be to ensure the correct conduct of the study in each centre, the collection and recording of data generated in writing, its documentation, recording and reporting, in accordance with the legislative and regulatory provisions in force. Monitoring reports will ensure traceability.

\subsection{Trial Status}

The APACAN2 trial is currently recruiting. Participant recruitment began in April 2018 and is expected to finish in December 2022. The approved protocol is version 8 , dated 24/02/2021.

\section{DISCUSSION}

The APACAN2 trial aims to assess the feasibility of a homebased adapted physical activity program during neoadjuvant chemotherapy for HER2-positive breast cancer. In to this intervention, we aim to encourage physical activity from the beginning of the treatment by proposing a physical activity program at home and without time constraints. In addition, we focus on a population that can draw particular benefit from the practice of physical activity because of the treatment-induced cardiotoxity to which HER2-positive patients are exposed.

The current standard care to monitor cardiotoxicity is left ventricular ejection fraction (LVEF) monitoring (40-42). However, this method has some limits, as it lacks sensitivity, and does not enable early detection of cardiotoxicity. New techniques are currently developing, such as the titration of troponin in the blood. This biomarker is recognized as predictive of cardiotoxicity occurrence during treatment by the Food and Drug Administration and in several studies because it precedes decreases in LVEF (4346). It would be interesting to follow levels of biomarkers such as troponin for the patients participating in the study.

We have chosen not to use a randomized design because it is a feasibility study and it was important that as many patients as possible should benefit from the intervention. According to the APACAN2 results, we could subsequently propose a randomized study. Another limit of our study is that the anthropometric measures and lipid test are done when the patient come for her consultation so they are not always fasting.

The strength of our study is that the physical activity program is proposed at the beginning of the treatment, as recommended. In the literature, the majority of studies deploy the physical activity program in the course of adjuvant chemotherapy or later. There is little data about physical activity and sedentariness during neoadjuvant chemotherapy for breast cancer. Furthermore, the home-based concept makes it possible to take geographical and temporal constraints into account. Indeed, patients are free to organise their sessions as they wish without complying with a specific imposed calendar, and they are not obliged to travel to a given place to train. 


\section{ETHICS STATEMENT}

The study protocol and its amendments has obtained approval from the French Ethics Committee (Comite de Protection des Personnes Sud-Est VI) (NID RCB 2016-A01344-47) in November 2016. The study is conducted in accordance with the Helsinki Declaration, the Good Clinical Practice (GCP) guidelines of the International Conference on Harmonisation (ICH-E6, 17/07/96).

\section{AUTHOR CONTRIBUTIONS}

$\mathrm{AG}, \mathrm{IM}, \mathrm{ET}$ and $\mathrm{XD}$ wrote the protocol. $\mathrm{XD}$ is the coordinator of the study. IM is the statistical expert, contributed to sample size

\section{REFERENCES}

1. Bray F, Ferlay J, Soerjomataram I, Siegel RL, Torre LA, Jemal A. Global Cancer Statistics 2018: GLOBOCAN Estimates of Incidence and Mortality Worldwide for 36 Cancers in 185 Countries. CA Cancer J Clin (2018) 68:394424. doi: 10.3322/caac.21492

2. Cobleigh MA, Vogel CL, Tripathy D, Robert NJ, Scholl S, Fehrenbacher L, et al. Multinational Study of the Efficacy and Safety of Humanized Anti-HER2 Monoclonal Antibody in Women Who Have HER2-Overexpressing Metastatic Breast Cancer That has Progressed After Chemotherapy for Metastatic Disease. J Clin Oncol (1999) 17:2639-48. doi: 10.1200/ JCO.1999.17.9.2639

3. Slamon DJ, Clark GM, Wong SG, Levin WJ, Ullrich A, McGuire WL. Human Breast Cancer: Correlation of Relapse and Survival With Amplification of the HER-2/Neu Oncogene. Science (1987) 235:177-82. doi: 10.1126/ science. 3798106

4. Moja L, Tagliabue L, Balduzzi S, Parmelli E, Pistotti V, Guarneri V, et al. Trastuzumab Containing Regimens for Early Breast Cancer. Cochrane Database Syst Rev (2012) CD006243. doi: 10.1002/14651858.CD006243.pub2

5. Mantarro S, Rossi M, Bonifazi M, D’Amico R, Blandizzi C, La Vecchia C, et al. Risk of Severe Cardiotoxicity Following Treatment With Trastuzumab: A Meta-Analysis of Randomized and Cohort Studies of 29,000 Women With Breast Cancer. Intern Emerg Med (2016) 11:123-40. doi: 10.1007/ s11739-015-1362-x

6. Chen T, Xu T, Li Y, Liang C, Chen J, Lu Y, et al. Risk of Cardiac Dysfunction With Trastuzumab in Breast Cancer Patients: A Meta-Analysis. Cancer Treat Rev (2011) 37:312-20. doi: 10.1016/j.ctrv.2010.09.001

7. Slamon DJ, Leyland-Jones B, Shak S, Fuchs H, Paton V, Bajamonde A, et al. Use of Chemotherapy Plus a Monoclonal Antibody Against HER2 for Metastatic Breast Cancer That Overexpresses HER2. N Engl J Med (2001) 344:783-92. doi: 10.1056/NEJM200103153441101

8. Sengupta PP, Northfelt DW, Gentile F, Zamorano JL, Khandheria BK. Trastuzumab-Induced Cardiotoxicity: Heart Failure at the Crossroads. Mayo Clin Proc (2008) 83:197-203. doi: 10.4065/83.2.197

9. Farolfi A, Melegari E, Aquilina M, Scarpi E, Ibrahim T, Maltoni R, et al. Trastuzumab-Induced Cardiotoxicity in Early Breast Cancer Patients: A Retrospective Study of Possible Risk and Protective Factors. Heart (2013) 99:634-9. doi: 10.1136/heartjnl-2012-303151

10. Fried G, Regev T, Moskovitz M. Trastuzumab-Related Cardiac Events in the Treatment of Early Breast Cancer. Breast Cancer Res Treat (2013) 142:1-7. doi: 10.1007/s10549-013-2732-6

11. Thavendiranathan P, Abdel-Qadir H, Fischer HD, Camacho X, Amir E, Austin PC, et al. Breast Cancer Therapy-Related Cardiac Dysfunction in Adult Women Treated in Routine Clinical Practice: A Population-Based Cohort Study. J Clin Oncol (2016) 34:2239-46. doi: 10.1200/JCO.2015.65.1505

12. McArthur HL, Chia S. Cardiotoxicity of Trastuzumab in Clinical Practice. N Engl J Med (2007) 357:94-5. doi: 10.1056/NEJMc070065 calculations and developed the experimental plan. She will undertake the statistical analyses. MD supervised the physical activity evaluation. AG is the project manager of the study and is involved in aspects of the day-to-day running of the trial. She wrote the first draft of this manuscript and contributed to the grant proposal. All authors critically revised the manuscript, gave final approval of the manuscript and are accountable for the accuracy and integrity of the manuscript.

\section{FUNDING}

This work received funding from the Quality of life Pink Ribbon Award by the association "Ruban Rose" in 2019 (www. cancerdusein.org).

13. Carayol M, Bernard P, Boiché J, Riou F, Mercier B, Cousson-Gélie F, et al Psychological Effect of Exercise in Women With Breast Cancer Receiving Adjuvant Therapy: What is the Optimal Dose Needed? Ann Oncol (2013) 24:291-300. doi: 10.1093/annonc/mds342

14. Furmaniak AC, Menig M, Markes MH. Exercise for Women Receiving Adjuvant Therapy for Breast Cancer. Cochrane Database Syst Rev (2016) 9: CD005001. doi: 10.1002/14651858.CD005001.pub3

15. Invernizzi M, de Sire A, Venetis K, Cigna E, Carda S, Borg M, et al. Quality of Life Interventions in Breast Cancer Survivors: State of the Art in Targeted Rehabilitation Strategies. Anticancer Agents Med Chem (2021) 21. doi: 10.2174/1871520621666210609095602

16. Zeng Y, Huang M, Cheng ASK, Zhou Y, So WKW. Meta-Analysis of the Effects of Exercise Intervention on Quality of Life in Breast Cancer Survivors. Breast Cancer (2014) 21:262-74. doi: 10.1007/s12282-014-0521-7

17. Kim C-J, Kang D-H, Smith BA, Landers KA. Cardiopulmonary Responses and Adherence to Exercise in Women Newly Diagnosed With Breast Cancer Undergoing Adjuvant Therapy. Cancer Nurs (2006) 29:156-65. doi: 10.1097/ 00002820-200603000-00013

18. Cornette T, Vincent F, Mandigout S, Antonini MT, Leobon S, Labrunie A, et al. Effects of Home-Based Exercise Training on VO2 in Breast Cancer Patients Under Adjuvant or Neoadjuvant Chemotherapy (SAPA): A Randomized Controlled Trial. Eur J Phys Rehabil Med (2016) 52:223-32.

19. Foucaut A-M, Berthouze-Aranda SE, Touillaud M, Kempf-Lépine A-S, Baudinet C, Meyrand R, et al. Reduction of Health Risk Factors Through an Adapted Physical Activity Program in Patients With Breast Cancer. Support Care Cancer (2014) 22:1097-104. doi: 10.1007/s00520-013-2065-3

20. Leach HJ, Danyluk JM, Nishimura KC, Culos-Reed SN. Benefits of 24 Versus 12 Weeks of Exercise and Wellness Programming for Women Undergoing Treatment for Breast Cancer. Support Care Cancer (2016) 24:4597-606. doi: 10.1007/s00520-016-3302-3

21. Blanchard CM, Courneya KS, Stein K. American Cancer Society's SCS-II. Cancer Survivors' Adherence to Lifestyle Behavior Recommendations and Associations With Health-Related Quality of Life: Results From the American Cancer Society's SCS-II. J Clin Oncol (2008) 26:2198-204. doi: 10.1200/ JCO.2007.14.6217

22. Coups EJ. Ostroff JS. A Population-Based Estimate of the Prevalence of Behavioral Risk Factors Among Adult Cancer Survivors and Noncancer Controls. Prev Med (2005) 40:702-11. doi: 10.1016/j.ypmed.2004.09.011

23. Eakin EG, Youlden DR, Baade PD, Lawler SP, Reeves MM, Heyworth JS, et al. Health Behaviors of Cancer Survivors: Data From an Australian PopulationBased Survey. Cancer Causes Control (2007) 18:881-94. doi: 10.1007/s10552007-9033-5

24. Harrison S, Hayes SC, Newman B. Level of Physical Activity and Characteristics Associated With Change Following Breast Cancer Diagnosis and Treatment. Psychooncology (2009) 18:387-94. doi: 10.1002/pon.1504

25. Turner RR, Steed L, Quirk H, Greasley RU, Saxton JM, Taylor SJ, et al. Interventions for Promoting Habitual Exercise in People Living With and 
Beyond Cancer. Cochrane Database Syst Rev (2018) 9:CD010192. doi: 10.1002/14651858.CD010192.pub3

26. WHO. Global Recommendations on Physical Activity for Health (2010). Geneva: World Health Organization. Available at: http://www.ncbi.nlm.nih. gov/books/NBK305057/ (Accessed May 7, 2018).

27. Ginzac A, Passildas J, Gadéa E, Abrial C, Molnar I, Trésorier R, et al. Treatment-Induced Cardiotoxicity in Breast Cancer: A Review of the Interest of Practicing a Physical Activity. Oncology (2019) 96:223-34. doi: $10.1159 / 000499383$

28. Haykowsky MJ, Mackey JR, Thompson RB, Jones LW, Paterson DI. Adjuvant Trastuzumab Induces Ventricular Remodeling Despite Aerobic Exercise Training. Clin Cancer Res (2009) 15:4963-7. doi: 10.1158/1078-0432.CCR09-0628

29. Hornsby WE, Douglas PS, West MJ, Kenjale AA, Lane AR, Schwitzer ER, et al. Safety and Efficacy of Aerobic Training in Operable Breast Cancer Patients Receiving Neoadjuvant Chemotherapy: A Phase II Randomized Trial. Acta Oncol (2014) 53:65-74. doi: 10.3109/0284186X.2013.781673

30. Jacquinot Q, Meneveau N, Chatot M, Bonnetain F, Degano B, Bouhaddi M, et al. A Phase 2 Randomized Trial to Evaluate the Impact of a Supervised Exercise Program on Cardiotoxicity at 3 Months in Patients With HER2 Overexpressing Breast Cancer Undergoing Adjuvant Treatment by Trastuzumab: Design of the CARDAPAC Study. BMC Cancer (2017) 17:425. doi: 10.1186/s12885-017-3420-4

31. Foucaut A-M, Berthouze SE, Touillaud M, Morelle M, Bourne-Branchu V, Kempf-Lépine A-S, et al. Deterioration of Physical Activity Level and Metabolic Risk Factors After Early-Stage Breast Cancer Diagnosis. Cancer Nurs (2015) 38:E1-9. doi: 10.1097/NCC.0000000000000187

32. Holick CN, Newcomb PA, Trentham-Dietz A, Titus-Ernstoff L, Bersch AJ, Stampfer MJ, et al. Physical Activity and Survival After Diagnosis of Invasive Breast Cancer. Cancer Epidemiol Biomarkers Prev (2008) 17:379-86. doi: 10.1158/1055-9965.EPI-07-0771

33. Irwin ML, McTiernan A, Manson JE, Thomson CA, Sternfeld B, Stefanick ML, et al. Physical Activity and Survival in Postmenopausal Women With Breast Cancer: Results From the Women's Health Initiative. Cancer Prev Res (Phila) (2011) 4:522-9. doi: 10.1158/1940-6207.CAPR-10-0295

34. Golubic R, May AM, Benjaminsen Borch K, Overvad K, Charles M-A, Diaz MJT, et al. Validity of Electronically Administered Recent Physical Activity Questionnaire (RPAQ) in Ten European Countries. PloS One (2014) 9:e92829. doi: 10.1371/journal.pone.0092829

35. Aaronson NK, Ahmedzai S, Bergman B, Bullinger M, Cull A, Duez NJ, et al. The European Organization for Research and Treatment of Cancer QLQ-C30: A Quality-of-Life Instrument for Use in International Clinical Trials in Oncology. J Natl Cancer Inst (1993) 85:365-76. doi: 10.1093/jnci/ 85.5.365

36. Gentile S, Delarozière JC, Favre F, Sambuc R, San Marco JL. Validation of the French "Multidimensional Fatigue Inventory" (MFI 20). Eur J Cancer Care (Engl) (2003) 12:58-64. doi: 10.1046/j.1365-2354.2003.00295.x

37. Ainsworth BE, Haskell WL, Whitt MC, Irwin ML, Swartz AM, Strath SJ, et al. Compendium of Physical Activities: An Update of Activity Codes and MET Intensities. Med Sci Sports Exerc (2000) 32:S498-504. doi: 10.1097/00005768200009001-00009
38. ATS Committee on Proficiency Standards for Clinical Pulmonary Function Laboratories. ATS Statement: Guidelines for the Six-Minute Walk Test. Am J Respir Crit Care Med (2002) 166:111-7. doi: 10.1164/ajrccm.166.1.at1102

39. Arcuri JF, Borghi-Silva A, Labadessa IG, Sentanin AC, Candolo C, Pires Di Lorenzo VA. Validity and Reliability of the 6-Minute Step Test in Healthy Individuals: A Cross-Sectional Study. Clin J Sport Med (2016) 26:69-75. doi: 10.1097/JSM.0000000000000190

40. Chavez-MacGregor M, Niu J, Zhang N, Elting LS, Smith BD, Banchs J, et al. Cardiac Monitoring During Adjuvant Trastuzumab-Based Chemotherapy Among Older Patients With Breast Cancer. J Clin Oncol (2015) 33:2176-83. doi: 10.1200/JCO.2014.58.9465

41. Senkus E, Kyriakides S, Ohno S, Penault-Llorca F, Poortmans P, Rutgers E, et al. Primary Breast Cancer: ESMO Clinical Practice Guidelines for Diagnosis, Treatment and Follow-Up. Ann Oncol (2015) 26(Suppl 5):v8-30. doi: 10.1093/annonc/mdv298

42. Jerusalem G, Lancellotti P, Kim S-B. HER2+ Breast Cancer Treatment and Cardiotoxicity: Monitoring and Management. Breast Cancer Res Treat (2019) 177:237-50. doi: 10.1007/s10549-019-05303-y

43. Kitayama H, Kondo T, Sugiyama J, Kurimoto K, Nishino Y, Kawada M, et al. High-Sensitive Troponin T Assay can Predict Anthracycline- and Trastuzumab-Induced Cardiotoxicity in Breast Cancer Patients. Breast Cancer (2017) 24:774-82. doi: 10.1007/s12282-017-0778-8

44. Ky B, Putt M, Sawaya H, French B, Januzzi JL, Sebag IA, et al. Early Increases in Multiple Biomarkers Predict Subsequent Cardiotoxicity in Patients With Breast Cancer Treated With Doxorubicin, Taxanes, and Trastuzumab. J Am Coll Cardiol (2014) 63:809-16. doi: 10.1016/j.jacc.2013.10.061

45. Wallace KB, Hausner E, Herman E, Holt GD, MacGregor JT, Metz AL, et al. Serum Troponins as Biomarkers of Drug-Induced Cardiac Toxicity. Toxicol Pathol (2004) 32:106-21. doi: 10.1080/01926230490261302

46. Mokuyasu S, Suzuki Y, Kawahara E, Seto T, Tokuda Y. High-Sensitivity Cardiac Troponin I Detection for 2 Types of Drug-Induced Cardiotoxicity in Patients With Breast Cancer. Breast Cancer (2015) 22:563-9. doi: 10.1007/ s12282-014-0520-8

Conflict of Interest: The authors declare that the research was conducted in the absence of any commercial or financial relationships that could be construed as a potential conflict of interest.

Publisher's Note: All claims expressed in this article are solely those of the authors and do not necessarily represent those of their affiliated organizations, or those of the publisher, the editors and the reviewers. Any product that may be evaluated in this article, or claim that may be made by its manufacturer, is not guaranteed or endorsed by the publisher.

Copyright $\odot 2021$ Ginzac, Bernadach, Molnar, Duclos, Thivat and Durando. This is an open-access article distributed under the terms of the Creative Commons Attribution License (CC BY). The use, distribution or reproduction in other forums is permitted, provided the original author(s) and the copyright owner(s) are credited and that the original publication in this journal is cited, in accordance with accepted academic practice. No use, distribution or reproduction is permitted which does not comply with these terms. 